\title{
A Hydrophobic Surface Based on a Ni-P-PTFE Coating on a Metallic Bipolar Plate
}

Chien-Hung Lin ${ }^{1, *}$, Jia-Ren Lee ${ }^{2}$, Pei-Jing Teng ${ }^{1}$, Sung-Ying Tsai ${ }^{3}$,Hung-Hua Sheu ${ }^{4}$, Kung-Hsu Hou ${ }^{5}$, Ming-Der Ger

${ }^{1}$ Department of Physics, ROC Military Academy, Feng-Shan, Kaohsiung 830, Taiwan

${ }^{2}$ Department of Physics, National Kaohsiung Normal University, Kaohsiung, Taiwan

${ }^{3}$ Materials and Electro-optics Research Division, Chung-Shan Institute of Science and Technology

Tao-Yuan 335, Taiwan, ROC

${ }^{4}$ Department of Chemical and Materials Engineering, National Defense University, Taoyuan, 335

Taiwan

${ }^{5}$ Department of Power Vehicle and Systems Engineering, National Defense University, Taoyuan, 335

Taiwan

*E-mail: linhungcma@gmail.com

doi: $10.20964 / 2018.03 .12$

Received: 11 May 2017 / Accepted: 29 July 2017 / Published: 5 February 2018

The performance of 5052 aluminum bipolar plates (BPPs) with surface treatment for proton exchange membrane fuel cell (PEMFC) systems are characterized in this study. The coated bipolar plates are evaluated by the coating composition, contact angle, corrosion resistance, interfacial contact resistance and assembly test. The coated bipolar plate has active water-management ability due to the Ni-P-PTFE (polytetrafluoroethylene) coating layer with low surface energy. The extremely hydrophobic property enhances the contact angle on the solid surface from $72^{\circ}$ to $103^{\circ}$. Furthermore, the corrosion resistance of the Ni-P-PTFE and substrate in the simulated environment are $1.02 \times 10^{-6}$ and $9.22 \times 10^{-4} \mathrm{~A} / \mathrm{cm}^{2}$, respectively. Hence, the substrate with a Ni-P-PTFE coating retains its superior performance after a durability test. The result demonstrates that the whole performance of the PEMFC is directly related to the corrosion resistance and hydrophobic behaviour on 5052 aluminum bipolar plate.

Keywords: proton exchange membrane fuel cell (PEMFC), Ni-P-PTFE coating, corrosion, hydrophobic, water-management

\section{$\underline{\text { FULL TEXT }}$}

(C) 2018 The Authors. Published by ESG (www.electrochemsci.org). This article is an open access article distributed under the terms and conditions of the Creative Commons Attribution license (http://creativecommons.org/licenses/by/4.0/). 